\title{
SURFACE DISPLACEMENT FIELD OF A COATED ELASTIC HALF-SPACE UNDER THE INFLUENCE OF A MOVING DISTRIBUTIONAL LOAD
}

\author{
Onur ŞAHIN ${ }^{1 *}$, Nihal EGE ${ }^{2}$ \\ ${ }^{1}$ Department of Mathematics, ) DFXOWIRICFIHFHHDQG\$ UWGiresun University, Giresun, Turkey \\ ${ }^{2}$ Department of Mathematics, ) DFXOMIRIC6FIHQFHПAnadolu University, Eskişehir, Turkey
}

\begin{abstract}
An analysis of the distributed moving load along the surface of a coated half space is presented. The formulation of the problem depends on the hyperbolic-elliptic asymptotic model developed earlier by the authors. The integral solution of the longitudinal and transverse displacements along the surface for the sub and super-Rayleigh cases are obtained by using the uniform stationary phase method. Numerical comparisons of the exact and asymptotic solutions of the longitudinal displacement are illustrated for the certain cross-sections of the profile.
\end{abstract}

Keywords: 3D elasticity, Asymptotic model, Moving load, Surface wave, Thin layer

\section{HAREKETLİ DAĞILIMLI YÜK ETKISİ ALTINDA KAPLAMALI BİR ELASTIKK YARI UZAYIN YÜZEY YER DEĞIŞTIRME ALANI}

\section{ÖZET}

Kaplamalı elastik bir yarı uzayın yüzeyi boyunca dağılımlı hareketli yükün bir analizi sunulmuştur. Problemin formülasyonu yazarlar tarafindan önceden geliştirilmiş hiperbolik-eliptik modele dayanmaktadır. Sub ve süper Rayleigh durumları için yüzey boyunca boyuna ve enine yer değiştirmelerin integral çözümleri düzgün durağan faz metodu kullanılarak elde edilmiştir. Boyuna yer değiştirmenin tam ve asimptotik çözümlerinin nümerik karşılaştırmaları profilin belirli kesitleri için grafikler ile gösterilmiştir.

Anahtar Kelimeler: 3-Boyutlu elastisite, Asimptotik model, Hareketli yük, Yüzey dalgası, İnce kaplama

\section{INTRODUCTION}

The propagation of surface waves in elastic structures under the action of moving loads is an active area of research. It has received significant attention due to its applicability in modern engineering application ranging from dynamic response of bridges, [1,2], to the effect of high-speed vehicles to the surrounding environments (see e.g., [3,4]). Most of these problems are modelled through a twodimensional (2D) setting, however real life problems require modelling and analysis of problems in a three-dimensional (3D) framework. Most of the works dealing with 3D problems either employ a numerical approach or leave the obtained solutions in integral forms both of which do not immediately yield to further physical analysis (a very good account of such solutions may be found in the leading texts by Achenbach [5] and Miklowitz [6]). These considerations necessitate different approaches one of which, the asymptotic approximation, is the essential theme of the current paper. The method used in this article, first introduced by Kaplunov et al. [7], relies on the relation of the longitudinal and transverse wave potentials (see eqn. (5), also Chadwick [8]) reducing the 3D problem to a pair of 2D plain problems. The model is also aimed at deriving the contribution arising from the Rayleigh surface wave. This allows one to analyse the physical parameters of the problem in a more straightforward manner.

*Corresponding Author: onur.sahin@giresun.edu.tr 
In this paper the dynamic response of the surface of a coated elastic half-space to a distributional load moving with a velocity $c$ is considered. In Section 2, the mathematical model is presented through the governing equations and boundary conditions. The problem is then scaled with respect to the thickness of the coating as well as the load speed, being closer to the surface wave speed. In Sections 3 and 4, the surface dynamics is investigated for super and sub-Rayleigh load speeds respectively. In both sections, the displacements fields are presented for the different problem parameters. The conclusions and the discussions of the obtained results are given in Section 5.

\section{STATEMENT OF THE PROBLEM}

We consider a 3D elastic half-space coated by a thin layer of thickness $h$, see Figure 1 .

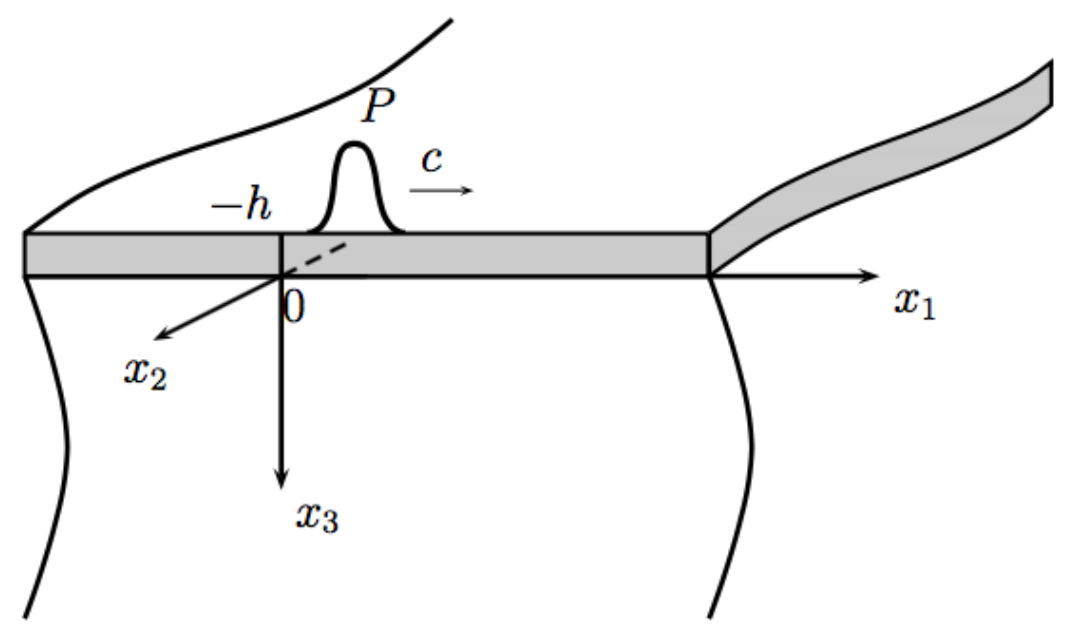

Figure 1. Coated half-space under the action of a moving distributional load

The equations of motion in 3D elasticity are adopted in their classical form [5]

$$
(\lambda+\mu) \operatorname{grad} \operatorname{div} \mathbf{u}+\mu \Delta \mathbf{u}=\rho \frac{\partial^{2} \mathbf{u}}{\partial t^{2}},
$$

where $\mathbf{u}=\left(\mathrm{u}_{1}, \mathrm{u}_{2}, \mathrm{u}_{3}\right)$ is the displacement vector, $\lambda$ and $\mu$ are Lamé constants, $\rho$ is the volume density and $\Delta$ is 3D Laplace operator. Here, the 3D coated elastic half-space is loaded with a distributional force of amplitude $P$, moving along the $O x_{1}$ axis on the surface at a constant speed $c$. The boundary conditions on the surface may hence be written as

$$
\begin{array}{cc}
\sigma_{i 3}=\frac{\partial u_{i}}{\partial x_{3}}+\frac{\partial u_{3}}{\partial x_{i}}=0, & x_{3}=-h, \\
\sigma_{33}=\lambda\left(\frac{\partial u_{1}}{\partial x_{1}}+\frac{\partial u_{2}}{\partial x_{2}}\right)+(\lambda+2 \mu) \frac{\partial u_{3}}{\partial x_{3}}=-P \frac{a}{\pi\left[\left(x_{1}-c t\right)^{2}+a^{2}\right]} \delta\left(x_{2}\right), & x_{3}=-h
\end{array}
$$

where $\sigma_{i 3}$ and $\sigma_{33}$ are components of the Cauchy stress tensor, $i=1,2, \delta(\cdot)$ is Dirac delta function and $a$ is a parameter describing the Gaussian profile of the load. Employing Helmholtz decomposition of a vector field (see, [9])

$$
\mathbf{u}=\operatorname{grad} \varphi+\operatorname{curl} \boldsymbol{\psi}
$$


and approximate hyperbolic-elliptic formulation presented in [7] and [10], we obtain pseudo-elliptic equations from equation (1) in the interior $-h<x_{3}$ of the half-space

$$
\frac{\partial^{2} \phi}{\partial x_{3}^{2}}+k_{1}^{2} \Delta_{2} \phi=0, \quad \frac{\partial^{2} \psi_{i}}{\partial x_{3}^{2}}+k_{2}^{2} \Delta_{2} \phi=0, \quad i=1,2
$$

with boundary equation at $x_{3}=0$

$$
\Delta_{2} \phi-\frac{1}{c_{R}^{2}} \frac{\partial^{2} \phi}{\partial t^{2}}-\frac{b h}{k_{1}} \frac{\partial}{\partial x_{3}}\left(\Delta_{2} \phi\right)=A P \frac{a}{\pi\left[\left(x_{1}-c t\right)^{2}+a^{2}\right]} \delta\left(x_{2}\right),
$$

and relations between the potentials

$$
\frac{\partial \phi}{\partial x_{i}}=\frac{2}{1+k_{2}^{2}} \frac{\partial \psi_{i}}{\partial x_{3}}, \quad i=1,2
$$

In the above formulae, the vector potential $\boldsymbol{\psi}$ is defined as $\boldsymbol{\psi}=\left(-\psi_{2}, \psi_{1}, 0\right), k_{i}=1-c_{R}^{2} / c_{i}^{2}$ are the wave numbers, $\Delta_{2}=\partial_{x_{1}}^{2}+\partial_{x_{2}}^{2}$ is the 2D Laplacian, $A$ and $b$ are constants first defined in [10], and $c_{R}$, $c_{1}$ and $c_{2}$ are the Rayleigh, longitudinal and transverse wave speeds respectively.

Solution of equation (3) may be written in symbolic form as

$$
\phi\left(x_{1}, x_{2}, x_{3}, t\right)=\exp \left(-k_{1} \sqrt{-\Delta_{2}} x_{3}\right) \phi\left(x_{1}, x_{2}, 0, t\right)
$$

where $\sqrt{-\Delta_{2}}$ is a pseudo differential operator (for further details see [10]). Straightforward differentiation of (6) gives

$$
\left.\frac{\partial \phi}{\partial x_{3}}\right|_{x_{3}=0}=-k_{1} \sqrt{-\Delta_{2}} \phi\left(x_{1}, x_{2}, 0, t\right)
$$

and equation (4) can be written as

$$
\Delta_{2} \phi-\frac{1}{c_{R}^{2}} \frac{\partial^{2} \phi}{\partial t^{2}}-b h \sqrt{-\Delta_{2}} \Delta_{2} \phi=A P \frac{a}{\pi\left[\left(x_{1}-c t\right)^{2}+a^{2}\right]} \delta\left(x_{2}\right) .
$$

It is a common practice that since the determination of the displacement field over the entire half-space is very difficult, if not impossible, throughout the paper we will focus on the surface displacements, i.e., displacements over $x_{3}=0$, which can be expressed through equations (2) and (5) in terms of one single potential as

$$
u_{i}=\frac{c_{R}^{2}}{2 c_{2}^{2}} \frac{\partial \phi}{\partial x_{i}}, \quad i=1,2
$$

We now restrict ourselves to steady-state regime with introducing moving coordinate $\chi=x_{1}-c t$. Rewriting equation (7) in terms of the new coordinate we get for the super-Rayleigh $\left(c>c_{R}\right)$ and the sub-Rayleigh $\left(c<c_{R}\right)$ regimes

$$
\frac{\partial^{2} \phi}{\partial x_{2}^{2}}-\varepsilon^{2} \frac{\partial^{2} \phi}{\partial \chi^{2}}-b h \sqrt{-\left(\frac{\partial^{2}}{\partial x_{2}^{2}}+\frac{\partial^{2}}{\partial \chi^{2}}\right)}\left(\frac{\partial^{2} \phi}{\partial x_{2}^{2}}+\frac{\partial^{2} \phi}{\partial \chi^{2}}\right)=A P \frac{a}{\pi\left[\chi^{2}+a^{2}\right]} \delta\left(x_{2}\right),
$$


and

$$
\frac{\partial^{2} \phi}{\partial x_{2}^{2}}+\varepsilon^{2} \frac{\partial^{2} \phi}{\partial \chi^{2}}-b h \sqrt{-\left(\frac{\partial^{2}}{\partial x_{2}^{2}}+\frac{\partial^{2}}{\partial \chi^{2}}\right)}\left(\frac{\partial^{2} \phi}{\partial x_{2}^{2}}+\frac{\partial^{2} \phi}{\partial \chi^{2}}\right)=A P \frac{a}{\pi\left[\chi^{2}+a^{2}\right]} \delta\left(x_{2}\right),
$$

respectively, where

$$
\varepsilon=\sqrt{1-\frac{c^{2}}{c_{R}^{2}}}
$$

The small parameter $\varepsilon$ signifies the fact that the speed of the applied load is close to the Rayleigh wave speed for which the approximate model is based on, so that $\varepsilon \ll 1$. Furthermore, thickness of the coating is taken smaller than a typical wave length (see, $[11,12])$. Here, it is also appropriate to expect that the distributions occur along the $x_{1}$ axis due to the direction of the movement of the load. Making use of the arguments mentioned above, it is reasonable to introduce the scaled variables

$$
\chi=\frac{\xi b h}{\varepsilon^{2}}, \quad x_{2}=\frac{\eta b h}{\varepsilon^{3}}
$$

On employing the scaling (11), equations (9) and (10) become, respectively,

$$
\frac{\partial^{2} \phi}{\partial \eta^{2}}-\frac{\partial^{2} \phi}{\partial \xi^{2}}-\sqrt{-\frac{\partial^{2}}{\partial \xi^{2}}} \frac{\partial^{2} \phi}{\partial \xi^{2}}=\frac{A P}{\varepsilon} \frac{a_{s}}{\pi\left[\xi^{2}+a_{s}^{2}\right]} \delta(\eta)
$$

and

$$
\frac{\partial^{2} \phi}{\partial \eta^{2}}+\frac{\partial^{2} \phi}{\partial \xi^{2}}-\sqrt{-\frac{\partial^{2}}{\partial \xi^{2}}} \frac{\partial^{2} \phi}{\partial \xi^{2}}=\frac{A P}{\varepsilon} \frac{a_{s}}{\pi\left[\xi^{2}+a_{s}^{2}\right]} \delta(\eta)
$$

where $a_{s}=a \varepsilon^{2} /(b h)$. It is the object of next two sections to obtain the solutions of equations (12) and (13).

\section{THE SUPER-RAYLEIGH REGIME}

Consider first the super-Rayleigh regime. On applying the Fourier transform to equation (12) in the variable $\xi$, we obtain

$$
\frac{d^{2} \phi^{F}}{d \eta^{2}}+k^{2}(1+|k|) \phi^{F}=\frac{A P}{\varepsilon} e^{-a_{s}|k|} \delta(\eta)
$$

where

$$
\phi^{F}(k, \eta, 0)=\int_{-\infty}^{\infty} \phi(\xi, \eta, 0) e^{-i k \xi} d \xi
$$

Similarly, employing the two-sided Laplace transform, defined as

$$
\phi^{F L}(k, s, 0)=\int_{-\infty}^{\infty} \phi(k, \eta, 0) e^{-s \eta} d \eta,
$$


to equation (14) we get

$$
s^{2} \phi^{F L}+k^{2}(1+|k|) \phi^{F L}=\frac{A P}{\varepsilon} e^{-a_{s}|k|},
$$

which results in

$$
\phi^{F L}=\frac{A P}{\varepsilon} \frac{1}{S^{2}+k^{2}(1+|k|)} e^{-a_{s}|k|} .
$$

The symmetry of the transformed potential with respect to $s$ enables the immediate use of Laplace transform tables (see, [13]) leading to

$$
\phi^{F}=\frac{A P}{\varepsilon} \frac{\sin (|k| \sqrt{1+|k|}|\eta|)}{|k| \sqrt{1+|k|}} e^{-a_{s}|k|} .
$$

The related inverse Fourier transform is then given by

$$
\begin{gathered}
\phi(\xi, \eta, 0)=\frac{A P}{2 \pi \varepsilon} \int_{-\infty}^{\infty} \frac{\sin (|k| \sqrt{1+|k|}|\eta|)}{|k| \sqrt{1+|k|}} e^{-a_{s}|k|} e^{i k \xi} d k \\
=\frac{A P}{\pi \varepsilon} \int_{0}^{\infty} \frac{\sin (k \sqrt{1+k}|\eta|) \cos (k \xi)}{k \sqrt{1+k}} e^{-a_{s} k} d k .
\end{gathered}
$$

The longitudinal and the transverse displacements, $u_{1}$ and $u_{2}$, may be expressed, through the new variables, on the surface $x_{3}=0$ as (cf. eqn (8))

$$
u_{1}=\frac{\varepsilon^{2} c_{R}^{2}}{2 b h c_{2}^{2}} \frac{\partial \phi}{\partial \xi}, u_{2}=\frac{\varepsilon^{3} c_{R}^{2}}{2 b h c_{2}^{2}} \frac{\partial \phi}{\partial \eta} .
$$

Let us first evaluate the longitudinal displacement $u_{1}$, which may be written through straightforward differentiation of (15) as

$$
\begin{aligned}
u_{1} & =-\frac{A P \varepsilon c_{R}^{2} \operatorname{sgn}(\xi)}{2 \pi b h c_{2}^{2}} \int_{0}^{\infty} \frac{\sin (k \sqrt{1+k}|\eta|) \sin (k|\xi|)}{\sqrt{1+k}} e^{-a_{s} k} d k \\
& =\frac{A P \varepsilon c_{R}^{2} \operatorname{sgn}(\xi)}{4 \pi b h c_{2}^{2}} \int_{0}^{\infty} \frac{\cos (k \sqrt{1+k}|\eta|+k|\xi|)-\cos (k \sqrt{1+k}|\eta|-k|\xi|)}{\sqrt{1+k}} e^{-a_{s} k} d k \\
& =\frac{A P \varepsilon c_{R}^{2} \operatorname{sgn}(\xi)}{4 \pi b h c_{2}^{2}} \operatorname{Re}\left\{\int_{0}^{\infty} \frac{e^{i|\xi|\left(k \sqrt{1+k} \mu_{*}+k\right)}}{\sqrt{1+k}} e^{-a_{s} k} d k-\int_{0}^{\infty} \frac{e^{i|\xi|\left(k \sqrt{1+k} \mu_{*}-k\right)}}{\sqrt{1+k}} e^{-a_{s} k} d k\right\},
\end{aligned}
$$

where $\mu_{*}=|\eta / \xi|$. 
Integrals appearing in eqn. (17) are frequently encountered in the dynamic surface wave propagation problems and in most cases, they are notoriously difficult to calculate if not impossible. We will therefore investigate the far-field asymptotic behaviour of the integrals as $|\xi| \gg 1$ and $\mu_{*} \sim 1$. It may be shown that while the first integral in (17) does not have any stationary points in the integration interval, i.e., any points for which the derivative vanishes, the second integral has one, which is obtained by taking the derivative of the exponent with respect to $k$, given by

$$
k_{*}=\frac{2\left(1-3 \mu_{*}^{2}+\sqrt{1+3 \mu_{*}^{2}}\right)}{9 \mu_{*}^{2}} .
$$

Consequently, the asymptotic contribution of the first integral in (17) is negligible in comparison to the second integral. It should also be noted that the stationary point $k_{*}$ coincides with the lower limit of the integral on the line of Mach cone $\mu_{*}=1$ (see, [11]). Therefore, we must use the uniform stationary phase method to obtain a uniform asymptotic expansion of the considered integral, (see [14,15]). Applying the uniform stationary phase method, we get at the leading order

$$
\begin{array}{r}
\int_{0}^{\infty} \frac{e^{i|\xi|\left(k \sqrt{1+k} \mu_{*}-k\right)}}{\sqrt{1+k}} e^{-a_{s} k} d k \sim f\left(k_{*}\right) \sqrt{\frac{2}{|\xi| h_{1}^{\prime \prime}\left(k_{*}\right)}}\left\{\cos \left(|\xi| h_{1}\left(k_{*}\right)\right)\left[\sqrt{\frac{\pi}{8}}-C\left(-k_{*} \sqrt{\frac{|\xi| h_{1}^{\prime \prime}\left(k_{*}\right)}{2}}\right)\right]\right. \\
\left.-\sin \left(|\xi| h_{1}\left(k_{*}\right)\right)\left[\sqrt{\frac{\pi}{8}}-S\left(-k_{*} \sqrt{\frac{|\xi| h_{1}^{\prime \prime}\left(k_{*}\right)}{2}}\right)\right]\right\}
\end{array}
$$

where

$$
h_{1}(k)=k \sqrt{1+k} \mu_{*}-k, \quad f(k)=\frac{e^{-a_{s} k}}{\sqrt{1+k}},
$$

and $C(x)$ and $S(x)$ are the Fresnel functions, defined by

$$
C(x)=\int_{0}^{x} \cos \left(t^{2}\right) d t, \quad S(x)=\int_{0}^{x} \sin \left(t^{2}\right) d t
$$

(see, [16]). Thus, the displacement $u_{1}$ is given by

$$
\begin{aligned}
u_{1} \sim-\frac{A P \varepsilon c_{R}^{2} \operatorname{sgn}(\xi)}{4 \pi b h c_{2}^{2}} f\left(k_{*}\right) \sqrt{\frac{2}{|\xi| h_{1}^{\prime \prime}\left(k_{*}\right)}} & \left\{\cos \left(|\xi| h_{1}\left(k_{*}\right)\right)\left[\sqrt{\frac{\pi}{8}}-C\left(-k_{*} \sqrt{\frac{|\xi| h_{1}^{\prime \prime}\left(k_{*}\right)}{2}}\right)\right]\right. \\
& \left.-\sin \left(|\xi| h_{1}\left(k_{*}\right)\right)\left[\sqrt{\frac{\pi}{8}}-S\left(-k_{*} \sqrt{\frac{|\xi| h_{1}^{\prime \prime}\left(k_{*}\right)}{2}}\right)\right]\right\}
\end{aligned}
$$

Following a similar approach for $u_{2}$ as in the preceding process, the far-field asymptotic expansion for the transverse displacement may be written as 


$$
\begin{array}{r}
u_{2} \sim-\frac{A P \varepsilon^{2} c_{R}^{2} \operatorname{sgn}(\eta)}{4 \pi b h c_{2}^{2}} g\left(k_{*}\right) \sqrt{\frac{2}{|\xi| h_{1}^{\prime \prime}\left(k_{*}\right)}}\left\{\operatorname { c o s } ( | \xi | h _ { 1 } ( k _ { * } ) ) \left[\sqrt{\frac{\pi}{8}}-C\left(-k_{*} \sqrt{\frac{|\xi| h_{1}^{\prime \prime}\left(k_{*}\right)}{2}}\right)\right.\right. \\
-\sin \left(|\xi| h_{1}\left(k_{*}\right)\right)\left[\sqrt{\frac{\pi}{8}}-S\left(-k_{*} \sqrt{\frac{|\xi| h_{1}^{\prime \prime}\left(k_{*}\right)}{2}}\right)\right]
\end{array}
$$

where

$$
g(k)=e^{-a_{s} k}
$$

In order to illustrate the accuracy of the approximate displacements we present below graphs of the comparisons of $u_{1}$ given by (18), and the exact solution (17), both of which are scaled as

$$
U_{1}=\frac{4 \pi b h c_{2}^{2}}{A P c_{R}^{2} \varepsilon} u_{1}
$$

In Figure 2, the longitudinal cross-sectional profile of the exact and asymptotic solutions of $U_{1}$ for different values of $a_{s}$ are presented. In this figure, solid lines correspond to exact solutions whereas the dashed lines correspond to asymptotic solutions. It is clearly seen in these graphs that the greater values of the parameter $a_{s}$ reduces the magnitude of the displacement resulting in a more uniform distributed profile, which is an expected result (cf. [17,18]). It should be noted that the values of the parameter $a_{s}$ either corresponds to the amplitude of the profile of the load for fixed values of the material parameter $b$ and the coating thickness $h$; or, to the coating thickness $h$ for fixed values of $a$, and $b$. This allows us to arbitrarily change either the profile of the load or the coating thickness according to the desired displacement outcome.

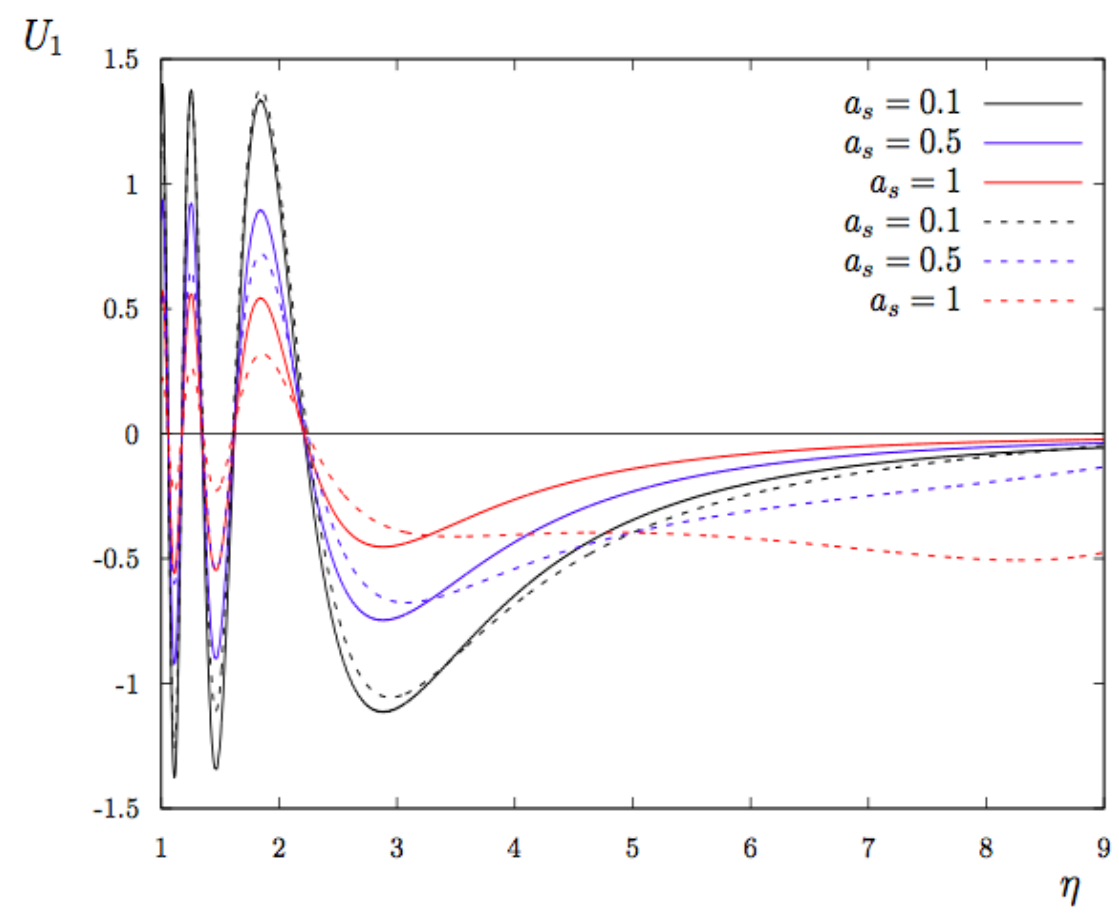

Figure 2. Super-Rayleigh displacement $U_{1}$ versus $\eta$ at $\xi=5$. 
Figure 3 compares the transverse cross-sectional profile of $U_{1}$ for exact and approximate solutions for different values of $a_{s}$. Once again the displacement amplitude reduces for larger values of $a_{s}$ demonstrating a smoothened displacement profile. We also would like to draw attention to the peculiar fact that, in both Figures 2 and 3 for increasing values of $a_{s}$, particularly for values greater than unity, the agreement between the exact and approximate solutions start to fail. This is not an inconsistency since the asymptotic solution is valid in the Mach cone $\mu_{*}=1(|\xi|=|\eta|$ ) (for further details see, $[11,12])$, and the argument of the exponential factor appearing in eqn. (18) becomes positive for certain particular values, making the displacement unbounded. However, these particular values are out of the Mach cone and therefore do not violate the validity of the obtained approximate displacement.

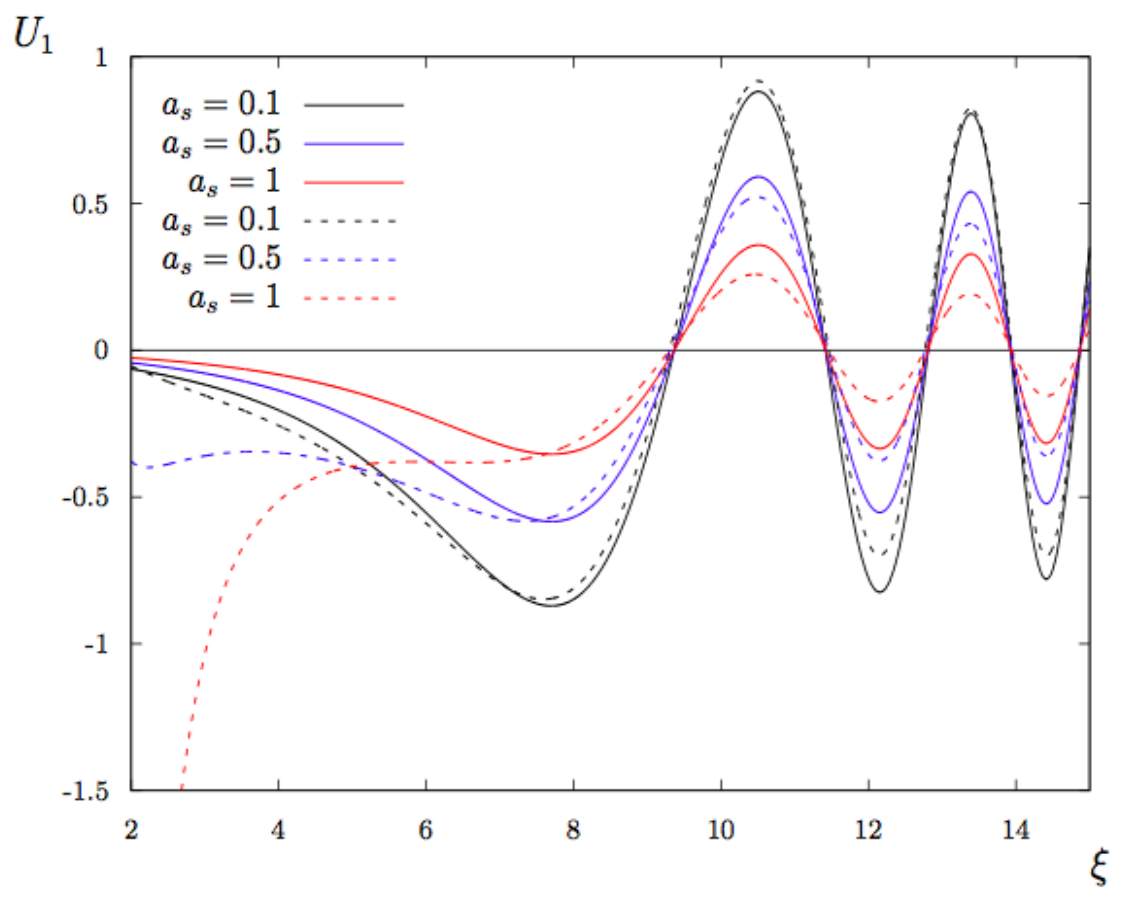

Figure 3. Super-Rayleigh displacement $U_{1}$ versus $\xi$ at $\eta=5$.

\section{THE SUB-RAYLEIGH REGIME}

We now proceed to the sub-Rayleigh regime. Taking the Fourier and Laplace transforms of equation (13) we obtain

$$
\phi^{F L}=\frac{A P}{\varepsilon} \frac{1}{S^{2}-k^{2}(1-|k|)} e^{-a_{s}|k|} .
$$

As before, the Laplace transform may easily be written using the symmetry of the potential in $s$, giving

$$
\phi^{F}(k, \eta, 0)=\left\{\begin{array}{cl}
-\frac{A P}{\varepsilon} \frac{e^{-|k|\left(\sqrt{1-|k|}|\eta|+a_{s}\right)}}{2|k| \sqrt{1-|k|}}, & |k|<1 \\
\frac{A P}{\varepsilon} \frac{e^{-a_{s}|k|} \sin (|k| \sqrt{|k|-1}|\eta|)}{|k| \sqrt{|k|-1}}, & |k|>1 .
\end{array}\right.
$$

Employing the inverse Fourier transform we arrive at 


$$
\begin{aligned}
\phi(k, \eta, 0)=\frac{A P}{\pi \varepsilon} & \left\{\int_{1}^{\infty} \frac{e^{-a_{s} k} \sin (k \sqrt{k-1}|\eta|)}{k \sqrt{k-1}} \cos (k|\xi|) d k\right. \\
& \left.-\int_{0}^{1} \frac{e^{-k\left(\sqrt{1-k}|\eta|+a_{s}\right)}}{2 k \sqrt{1-k}} \cos (k \xi) d k\right\} .
\end{aligned}
$$

The longitudinal surface displacement $u_{1}$ can be written from equation (16) in the following form

$$
\begin{aligned}
u_{1}(\xi, \eta, 0)= & \frac{A P \varepsilon c_{R}^{2} \operatorname{sgn}(\xi)}{2 \pi c_{2}^{2} b h}\left[\int_{0}^{1} \frac{e^{-k\left(\sqrt{1-k}|\eta|+a_{s}\right)}}{2 \sqrt{1-k}} \sin (k|\xi|) d k\right. \\
& \left.-\int_{1}^{\infty} \frac{e^{-a_{s} k} \sin \left(k \sqrt{k-1}|\xi| \mu_{*}\right)}{\sqrt{k-1}} \sin (k|\xi|) d k\right] .
\end{aligned}
$$

Considering the far-field approximation as $|\xi| \gg 1$, it may be shown that the leading order asymptotic behaviour of $u_{1}$ arises from the stationary point of the second integral in equation (19), which can be written as

$$
\begin{aligned}
\int_{1}^{\infty} \frac{\sin (k|\xi|) \sin \left(k \sqrt{k-1}|\xi| \mu_{*}\right)}{\sqrt{k-1}} e^{-a_{s} k} d k & =\frac{1}{2} \operatorname{Re}\left\{\int_{1}^{\infty} \frac{e^{i|\xi|\left(k \sqrt{k-1} \mu_{*}-k\right)}}{\sqrt{k-1}} e^{-a_{s} k} d k-\right. \\
& \left.-\int_{1}^{\infty} \frac{e^{i|\xi|\left(k \sqrt{k-1} \mu_{*}+k\right)}}{\sqrt{k-1}} e^{-a_{s} k} d k\right\} .
\end{aligned}
$$

A change of variable $t=\sqrt{k-1}$ transforms the integrals above to

$$
\begin{aligned}
& \int_{1}^{\infty} \frac{\sin (k|\xi|) \sin \left(k \sqrt{k-1}|\xi| \mu_{*}\right)}{\sqrt{k-1}} e^{-a_{s} k} d k=e^{-a_{s}} \operatorname{Re}\left\{\int_{0}^{\infty} e^{i|\xi|\left(\mu_{*} t^{3}-t^{2}+t \mu_{*}-1\right)} e^{-a_{s} t^{2}} d t-\right. \\
& \left.-\int_{0}^{\infty} e^{i|\xi|\left(\mu_{*} t^{3}+t^{2}+t \mu_{*}+1\right)} e^{-a_{s} t^{2}} d t\right\}
\end{aligned}
$$

If we analyse the stationary points of the integrals in eqn. (20), we immediately observe that only the first integral in (19) has stationary points which are given by

$$
t_{1}=\frac{1+\sqrt{1-3 \mu_{*}^{2}}}{3 \mu_{*}} \quad \text { and } \quad t_{2}=\frac{1-\sqrt{1-3 \mu_{*}^{2}}}{3 \mu_{*}}, \quad 0<\mu_{*} \leq 1 / \sqrt{3} .
$$


It should be emphasized that the two stationary points converge to each other along the line $\mu_{*}=$ $1 / \sqrt{3}$ which imposes the use of the stationary phase method. Thus, we are concerned with the approximate value of an integral of the form

$$
\int_{0}^{\infty} e^{i|\xi| h_{2}\left(t, \mu_{*}\right)} g(t) d t
$$

where, in our specific problem,

$$
h_{2}\left(t, \mu_{*}\right)=\mu_{*} t^{3}-t^{2}+\mu_{*} t-1 \text { and } g(t)=e^{-a_{s} t^{2}} .
$$

A change of variable of the form

$$
h_{2}\left(t, \mu_{*}\right)=\frac{u^{3}}{3}-\alpha u+\beta
$$

introduced by Chester et al. [19], provides a means of obtaining a uniform asymptotic expansion when two stationary points coincide. Here $\alpha$ and $\beta$ are determined as

$$
\alpha=\frac{1-3 \mu_{*}^{2}}{\left(3 \mu_{*}\right)^{4 / 3}}, \quad \beta=-\frac{2}{27}\left(9+\frac{1}{\mu_{*}^{2}}\right) .
$$

Substituting (22) into the integral in equation (21), we get

$$
e^{i|\xi| \beta} e^{-a_{s}} \int_{0}^{\infty} e^{i|\xi|\left(u^{3} / 3-\alpha u\right)} p(u) d u,
$$

where

$$
p(u)=e^{-a_{s} t^{2}} \frac{d t}{d u}
$$

and $t=t(u)$ is a single valued analytic function and derivation of $t(u)$ is given by

$$
\frac{d t}{d u}=\frac{u^{2}-\alpha}{h_{2}^{\prime}\left(t, \mu_{*}\right)_{t}}
$$

for more details see [15]. Following Bleistein [20], $p(u)$ can be written as

$$
p(u)=a_{0}+b_{0} u+\left(u^{2}-\zeta\right) r(u)
$$

where

and

$$
a_{0}=\frac{\alpha^{1 / 4}}{\sqrt{2}}\left[\frac{e^{-a_{s} t_{2}^{2}}}{\sqrt{h_{2}^{\prime \prime}\left(t_{2}\right)}}+\frac{e^{-a_{s} t_{1}^{2}}}{\sqrt{h_{2}^{\prime \prime}\left(t_{1}\right)}}\right]
$$




$$
b_{0}=-\frac{\alpha^{-1 / 4}}{\sqrt{2}}\left[\frac{e^{-a_{s} t_{2}^{2}}}{\sqrt{h_{2}^{\prime \prime}\left(t_{2}\right)}}-\frac{e^{-a_{s} t_{1}^{2}}}{\sqrt{h_{2}^{\prime \prime}\left(t_{1}\right)}}\right] \text {. }
$$

Thus, (23) is written as

$$
e^{i|\xi| \beta} e^{-a_{s}}\left\{a_{0} \int_{-\infty}^{\infty} e^{i|\xi|\left(u^{3} / 3-\alpha u\right)} p(u) d u+b_{0} \int_{-\infty}^{\infty} u e^{i|\xi|\left(u^{3} / 3-\alpha u\right)} d u+I(|\xi|, \alpha)\right\},
$$

where

$$
I(|\xi|, \alpha)=\int_{-\infty}^{\infty}\left(u^{2}-\zeta\right) e^{i|\xi|\left(u^{3} / 3-\alpha u\right)} r(u) d u .
$$

After applying the same process for $I(|\xi|, \alpha)$ it may be seen that the asymptotic contribution of $I(|\xi|, \alpha)$ is negligible in comparison to the integrals in (24). Therefore, the asymptotic expansion of the integral given in (20) takes the form

$$
\begin{gathered}
\int_{1}^{\infty} \frac{\sin (k|\xi|) \sin \left(k \sqrt{k-1}|\xi| \mu_{*}\right)}{\sqrt{k-1}} e^{-a_{s} k} d k \sim e^{-a_{s}} \operatorname{Re}\left\{e ^ { i | \xi | \beta } \left(2 \pi a_{0}|\xi|^{-1 / 3} \operatorname{Ai}\left(-|\xi|^{2 / 3} \alpha\right)-\right.\right. \\
\left.\left.-i 2 \pi b_{0}|\xi|^{-2 / 3} \operatorname{Ai}^{\prime}\left(-|\xi|^{2 / 3} \alpha\right)\right)\right\} .
\end{gathered}
$$

where $\operatorname{Ai}(x)$ is the Airy function, for more details see [16].

The resulting longitudinal displacement $u_{1}$ is then given by

$$
\begin{gathered}
u_{1} \sim-\frac{A P \varepsilon c_{\mathrm{R}}^{2} \operatorname{sgn}(\xi) e^{-a_{s}}}{b h c_{2}^{2}} \operatorname{Re}\left\{e ^ { i | \xi | \beta } \left(a_{0}|\xi|^{-1 / 3} \operatorname{Ai}\left(-|\xi|^{2 / 3} \alpha\right)\right.\right. \\
\left.\left.-i b_{0}|\xi|^{-2 / 3} \operatorname{Ai}^{\prime}\left(-|\xi|^{2 / 3} \alpha\right)\right)\right\}
\end{gathered}
$$

Obtaining the asymptotic expansion for the transverse displacement follows the same lines presented in detail for the longitudinal displacement, and therefore it is found as

$$
\begin{gathered}
u_{2} \sim-\frac{A P \varepsilon^{2} c_{\mathrm{R}}^{2} \operatorname{sgn}(\eta) e^{-a_{s}}}{b h c_{2}^{2}} \operatorname{Re}\left\{e ^ { i | \xi | \beta } \left(a_{1}|\xi|^{-1 / 3} \operatorname{Ai}\left(-|\xi|^{2 / 3} \alpha\right)\right.\right. \\
\left.\left.-i b_{1}|\xi|^{-2 / 3} \operatorname{Ai}^{\prime}\left(-|\xi|^{2 / 3} \alpha\right)\right)\right\}
\end{gathered}
$$

where

$$
a_{1}=\frac{\alpha^{1 / 4}}{\sqrt{2}}\left[\frac{t_{2} e^{-a_{s} t_{2}^{2}}}{\sqrt{h_{2}^{\prime \prime}\left(t_{2}\right)}}+\frac{t_{1} e^{-a_{s} t_{1}^{2}}}{\sqrt{h_{2}^{\prime \prime}\left(t_{1}\right)}}\right]
$$

and

$$
b_{1}=-\frac{\alpha^{-1 / 4}}{\sqrt{2}}\left[\frac{t_{2} e^{-a_{s} t_{2}^{2}}}{\sqrt{h_{2}^{\prime \prime}\left(t_{2}\right)}}-\frac{t_{1} e^{-a_{s} t_{1}^{2}}}{\sqrt{h_{2}^{\prime \prime}\left(t_{1}\right)}}\right] .
$$

The longitudinal displacement $U_{1}$ is scaled, similar as in Section 2, and written as 


$$
U_{1}=\frac{b h c_{2}^{2}}{A P c_{R}^{2} \varepsilon} .
$$

Figures 4 and 5 illustrate the comparisons of the exact and asymptotic displacements $U_{1}$ for the longitudinal and transverse cross sectional profiles, respectively. As before, the solid lines correspond to exact solutions and the dashed lines are the corresponding approximate solutions. Similar observations considered in the case of super Rayleigh regime apply here too, in that the smoothened displacement profiles require the values of the parameter $a_{s}$ to be large. However, care must be taken in the choice of the mentioned parameter taking into account the validity region (Mach cones) of the obtained asymptotic solutions. Therefore, in the graphs, although we see a diminished displacement profile in $U_{1}$ for the particular value $a_{s}=1$, the exponential factor in the asymptotic solution as well as the domain of validity of the approximation causes a discrepancy for smaller values of the variable $\eta$.

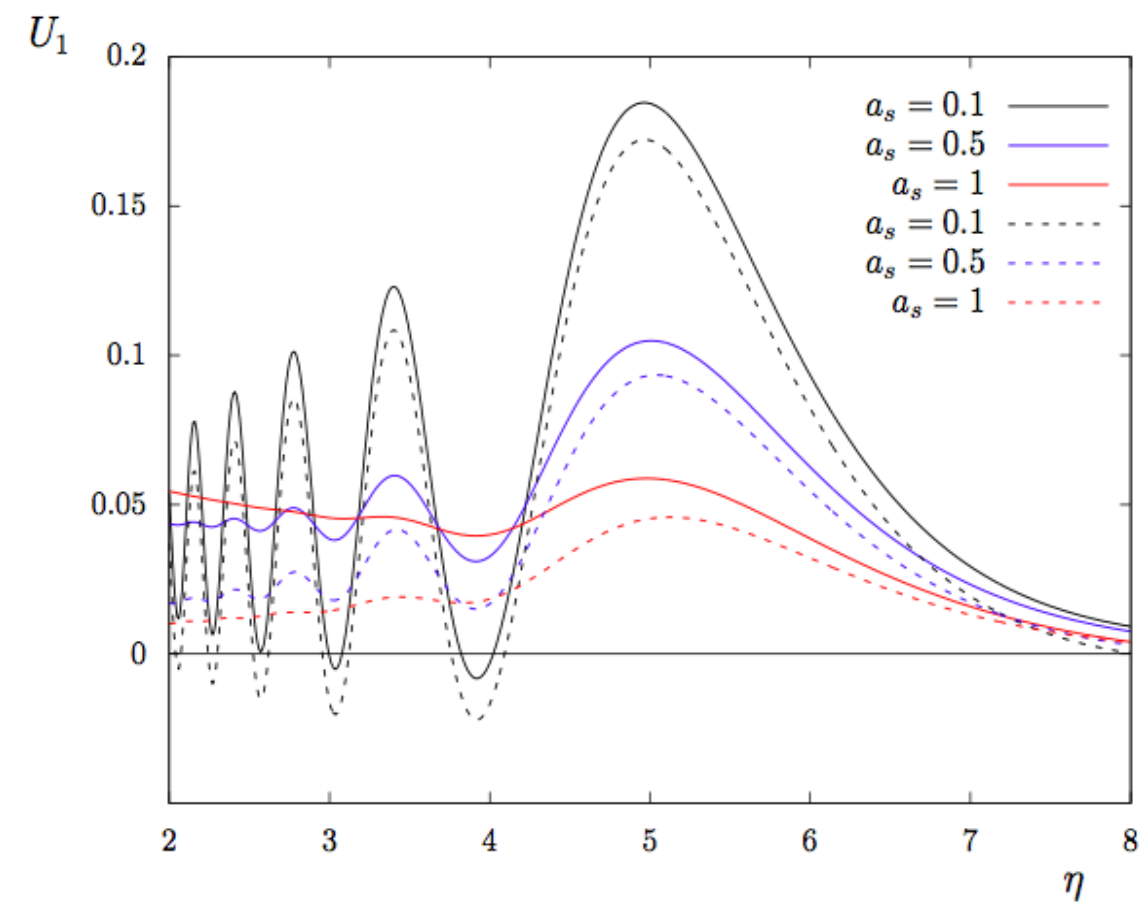

Figure 4. Sub-Rayleigh displacement $U_{1}$ versus $\eta$ at $\xi=10$. 


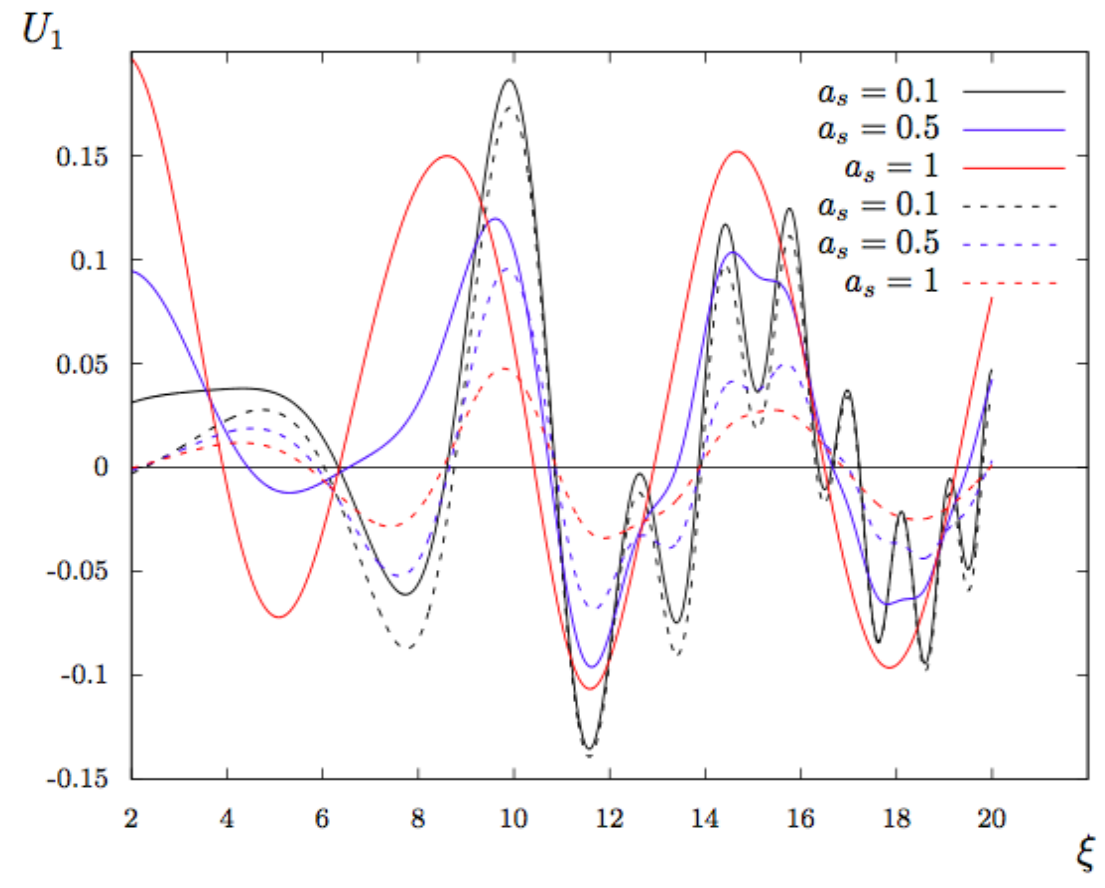

Figure 5. Sub-Rayleigh displacement $U_{1}$ versus $\xi$ at $\eta=5$.

\section{CONCLUSION}

In the present paper, the response of a 3D coated elastic half-space under the action of a distributed moving load is investigated. The displacement field is obtained through the application of an asymptotic model developed earlier by the authors. This approach reduces the $3 \mathrm{D}$ problem to a pair of $2 \mathrm{D}$ plain problems in the wave potentials, and therefore enables a convenient physical analysis of the original problem. The obtained approximate solutions for the displacement fields are expressed in terms of elementary functions differing from the known numerical solutions or solutions in terms of implicit integrals. This fact makes it possible to further examine the required elastic fields of the considered problem. The accuracy of the approximate solutions is presented by means of graphs giving comparisons of the asymptotic and exact solutions. It is clearly seen in the Figures that the effect of the coating and/or the width of the distributed load play a similar role reducing the singularity under the load encountered generally in point load problems. The parameter $a_{S}$ has a two-fold role: depending on the desired smoothness of the displacement field, either the thickness of the coating or the width of the distributed load may be altered, i.e. for a very thin layer, a larger width of the load might be chosen; if the layer thickness is large, even a point load may give smoothened results. Obviously, such choices are dictated by real-life applications and adjustments might be made according to the needs of the industry.

\section{REFERENCES}

[1] Zhu XQ and Law SS. Dynamic load on continuous multi-lane bridge deck from moving vehicles. Journal of Sound and Vibration 2002; 251: 697-716.

[2] Cao Y, Xia H and Li ZA. Semi-analytical/FEM model for predicting ground vibrations induced by high-speed train through continuous girder bridge. Journal of Mechanical Science and Technology 2012; 26: $2485-2496$.

[3] Agostinacchio M, Ciampa D, Diomedi M, and Olita S. Parametrical analysis of the railways dynamic response at high speed moving loads. J. Mod. Transp 2013; 21: 169-181. 
[4] El Kacimi A, Woodward PK, Laghrouche O, and Medero G. Time domain 3D finite element modelling of train-induced vibration at high speed. Comput. Struct. 2013; 118: 66-73.

[5] Achenbach JD. Wave propagation in elastic solids, North-Holland, Amsterdam, 1973.

[6] Miklowitz J. The theory of elastic waves and waveguides, Elsevier, 2012.

[7] Kaplunov J, Zakharov A and Prikazchikov DA. Explicit models for elastic and piezoelastic surface waves. IMA J. Appl. Math. 2006; 71: 768-782.

[8] Chadwick P. Surface and interfacial waves of arbitrary form in isotropic elastic media, J. of Elasticity 1976; 6: 73-80.

[9] Courant R and Hilbert D. Methods of Mathematical Physics, John Wiley \& Sons, 1989.

[10] Dai HH, Kaplunov J and Prikazchikov DA. A long-wave model for the surface elastic wave in a coated half-space. Proc. R. Soc. A. 2010; 466: 3097-3116.

[11] Kaplunov JD, Prikazchikov DA, Erbaş B, and Şahin O. On a 3D moving load problem for an elastic half space. Wave Motion 2013; 50: 1229-1238.

[12] Erbaş B, Kaplunov J, Prikazchikov DA and Şahin O. The near-resonant regimes of a moving load in a 3D problem for a coated elastic half space. Math. Mech. Solids 2014; doi: $10.1177 / 1081286514555451$.

[13] Debnath L, and Dambaru B. Integral transforms and their applications, CRC press, 2014.

[14] Borovikov VA. Uniform stationary phase method, Institution of Electrical Engineers, London, 1994.

[15] Wong R. Asymptotic Approximations of Integrals, Academic Press, 2014.

[16] Abramowitz M and Stegun IA. Handbook of mathematical functions, Dover Publications, New York, 2012.

[17] Erbaş B, Şahin O. On the causality of the Rayleigh wave. Journal of mechanics of materials and structures 2016, 11: 449-461.

[18] Erbaş B, Şahin O, Ege N. Response of a 3D elastic half-space to a distributed moving load. Hacettepe Journal of Mathematics and Statistics, in press.

[19] Chester C, Friedman B, and Ursell F. An extension of the method of steepest descents, Cambridge University Press, 1957.

[20] Bleistein N. Uniform asymptotic expansions of integrals with many nearby stationary points and algebraic singularities. Journal of Mathematics and Mechanics 1967; 17: 533-559. 